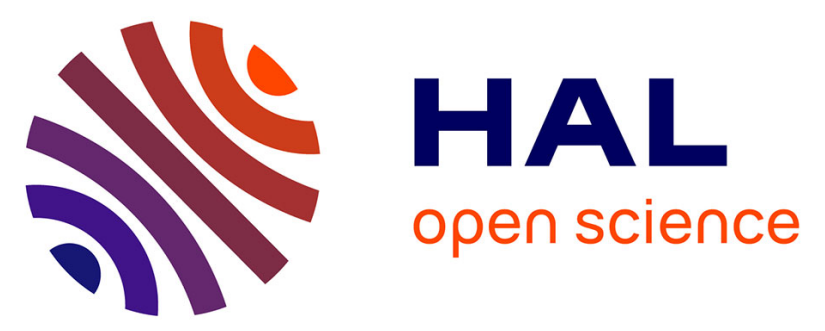

\title{
How far can the uncertainty on a Digital Soil Map be known?: A numerical experiment using pseudo values of clay content obtained from Vis-SWIR hyperspectral imagery
}

Philippe Lagacherie, Dominique Arrouays, Hocine Bourennane, Cécile Gomez, Manuel P. Martin, Nicolas P.A. Saby

\section{To cite this version:}

Philippe Lagacherie, Dominique Arrouays, Hocine Bourennane, Cécile Gomez, Manuel P. Martin, et al.. How far can the uncertainty on a Digital Soil Map be known?: A numerical experiment using pseudo values of clay content obtained from Vis-SWIR hyperspectral imagery. Geoderma, 2019, 337, pp.1320-1328. 10.1016/j.geoderma.2018.08.024 . hal-02058231

\section{HAL Id: hal-02058231 \\ https://hal.science/hal-02058231}

Submitted on 9 Sep 2021

HAL is a multi-disciplinary open access archive for the deposit and dissemination of scientific research documents, whether they are published or not. The documents may come from teaching and research institutions in France or abroad, or from public or private research centers.
L'archive ouverte pluridisciplinaire HAL, est destinée au dépôt et à la diffusion de documents scientifiques de niveau recherche, publiés ou non, émanant des établissements d'enseignement et de recherche français ou étrangers, des laboratoires publics ou privés. 
1 How far can the uncertainty on a Digital Soil Map be known? : a numerical experiment

2 using pseudo values of clay content obtained from Vis-SWIR Hyperspectral imagery

3 Philippe Lagacherie ${ }^{1}$, Dominique Arrouays ${ }^{2}$, Hocine Bourennane ${ }^{3}$, Cécile Gomez ${ }^{1}$, Manuel 4 Martin², Nicolas P.A. Saby $^{2}$

5

6 1. LISAH, Univ Montpellier, INRA, IRD, Montpellier SupAgro, Montpellier, France

7 2. Infosol, INRA, 45075 Orléans, France

8 3. UR Sols, INRA, 45075 Orléans, France

9

Corresponding author: Philippe Lagacherie, LISAH, INRA, 2 place Viala, 34060 Montpellier

11 (France). philippe.lagacherie@inra.fr

\section{Abstract}

14

Digital Soil Map uncertainty is usually evaluated from a set of independent soil observations

that are used to determine various uncertainty indicators. However, the number and locations

of the sites that constitute these evaluations may impact the value of these indicators.

In this paper, a numerical experiment on uncertainty indicators was performed using the pseudo values of topsoil clay content obtained from an airborne hyperspectral image in the

Cap Bon region (Tunisia). These pseudo values form a soil pattern with a large extent (46\% of

$\left.21300 \mathrm{~km}^{2}\right)$, high resolution $(5 \mathrm{~m})$ and good accuracy $\left(R^{2}\right.$ val $\left.=0.75\right)$ while being free of visible

22 artefacts and pedologically plausible. Therefore, the dataset was considered a fair representation of reality while providing a quasi-unlimited choice of sites. 
The numerical experiment considered three Quantile Regression Forests as examples of DSM models by using inputs from relief soil covariates and geographical locations that were calibrated from 200, 2,000 and 100,000 individuals respectively (low, medium and high quality models). Their uncertainty indicators were first evaluated by calculating four uncertainty indicators (ME, MSE, SS MSE and PICP) from a large independent validation set of 100,000 sites. These uncertainty indicators were then computed from independent evaluation sets of different sizes (from 50 to 500 sites) and from different locations (500 evaluation sets of each size). The independent evaluation sets were selected following a stratified random sampling using compact geographical strata.

The numerical experiment showed that the values of the uncertainty indicators were highly variable across numbers and locations of sites. The largest variations were observed for evaluation sets with fewer than 100 sites, but non-negligible variations remained for larger evaluation datasets. This result suggested that evaluations from independent sets convey a non-negligible error on the uncertainty indicators, which increases as the number of sites decrease.

Evaluations of DSM models from independent evaluation sets should be interpreted with care and uncertainty on validation results should be systematically estimated. For that, numerical experiments for benchmarking DSM models on known soil patterns across the world would be a valuable complement to the analytical expressions for the uncertainty indicators and the many DSM applications for which these analytical expressions are not valid. This would serve also to improve the sampling techniques for the calibration and evaluation datasets to reduce the error when estimating the uncertainty of a DSM product.

Keywords: Soil mapping, Uncertainty, Hyperspectral imagery, Random forest, Sampling 


\section{Introduction}

Soil maps are simplified representations of more complex and partially unknown patterns of soil variations. Therefore, any prediction of a soil property that can be derived from these soil

54 maps has an irreducible and often substantial error that is the difference between the true and estimated values of the soil property. Since there is no way to systematically measure this error, we are uncertain about the true value of the soil property at most of the locations, where uncertainty refers to the state of mind of a person who expresses a lack of confidence about reality (Heuvelink, 2014).

Getting accurate estimates of this uncertainty is of paramount importance for end-users to make enlightened decisions on the utility and limitations of the soil data products delivered to them. An example of a concrete translation of this exigence is provided by the GlobalSoilMap specifications (Arrouays et al., 2014). Following these specifications, each soil property estimate should be provided under the form of a $90 \%$ prediction interval (PI), which reports the range of values in which the true value is expected to occur 9 times out of 10 .

To provide this uncertainty information, a rigorous assessment of the uncertainty is considered a mandatory component of any Digital soil mapping application. Two main groups of methods can be used to accomplish this task (Heuvelink, 2014). The first is a model-based approach that involves spatial stochastic models that can provide estimates of the uncertainty of their own predictions. The main drawback of these model-based approaches is that their uncertainty evaluations are only valid under certain assumptions of the stochastic models. They also use the same data that were used to calibrate the models, which may cause an 
underestimation of the uncertainty. The second group of uncertainty evaluation methods avoids this restriction by undertaking a model-free statistical evaluation with independent sites selected by probability sampling. The comparison between the predicted and actual values of the soil attributes of interest yields a set of uncertainty indicators, the most common of which are the mean error (ME), the mean squared error (MSE), the root mean square error (RMSE) and the coefficient of determination $\left(R^{2}\right)$. The latter is confusingly referred to in the DSM literature either as the goodness-of-fit of a linear regression between the predicted and observed values, or to how close the paired prediction-observation points are to the $1: 1$ line. For DSM models that deliver predictions via probability distributions, accuracy plots (Goovaerts, 2001) or Prediction Interval Coverage probability (PICP) (Shrestha and Solomatine 2006), it can also be calculated to evaluate the accuracy of the uncertainty attached to each prediction.

Various methods for producing sets of independent sites (denoted further 'evaluation sets'), including the data-splitting of the available dataset, cross-validation over the available dataset and the probabilistic sampling of additional sites, were reviewed by Brus et al (2011). They recommended the latter to ensure unbiased uncertainty estimations and provide a more complete view of the spatial distribution of the error via the Spatial Cumulative Distribution Function (SCDF).

The main drawback of any model-free statistical evaluation is that the calculated uncertainty indicators are themselves prone to uncertainty. Indeed, similar to any statistical parameters that are derived from a set of individuals, the uncertainty indicators (e.g., $R^{2}, M E$, and PICP) are sensitive to the number and the locations of the soil observations used for calculating them. Under the condition that a probabilistic sampling is applied, Brus et al (2011) provided an analytical expression of the mean error (ME) and the mean square error (MSE) variances 
using the sampling fraction (the ratio between the number of samples and the number of possible sample locations) and the estimated variance of the error over a given area as inputs. Such analytical expressions of the ME and MSE variances measure the uncertainty of these uncertainty indicators, which could be very useful. For example, they could be used for determining whether the differences between the ME and MSE of two different DSM models reveal significantly different performances or for computing the size of the validation sample that is required for estimating the ME and MSE at a given precision level. However, it must be noted that i) for some indicators other than ME and MSE and ii) for some specific sampling designs (such as two-stage random sampling), there might not be readily available statistical estimates of the uncertainty of the uncertainty indicators (Brus et al. (2011)).

Although the uncertainty of the ME and MSE is easily computable for some sampling designs, it is far from being currently computed in DSM applications. This is because the soil datasets used as inputs of DSM models are rarely suitable for applying a probabilistic sampling. Indeed, these soil datasets are often undersized with regard to the size of the study area and the complexity of the soil cover to be modelled. This leads to substantial losses of predictive performances as soon as the sampling effort for collecting calibration sites is depleted for populating the set of independent sites required by probabilistic sampling. Furthermore, DSM applications most often use legacy data that do not respect the randomness and evenness required for a probabilistic sampling. For all these reasons, the DSM mappers most often disregard calculating any uncertainty in their uncertainty indicators and therefore neglect this issue when evaluating the DSM products.

This paper presents a numerical experiment for assessing the uncertainties of the uncertainty indicators of the three DSM models with contrasted predictive performances by using different probabilistic samplings of different sizes. To overcome the above-evoked limitations 
of the current soil input data, the study used the virtual pattern of the topsoil pseudo clay

121 content derived from airborne Vis-NIR-SWIR hyperspectral data acquired over the Cap Bon

122 region (300 km², Tunisia) at a five-meter resolution (Gomez et al., 2015). This pattern is

123 constituted of well-predicted clay values $\left(R^{2}=0.75\right)$ that are free of visible artefacts and

124 pedologically plausible, which allows it to be considered as a fair representation of the

125 variations of a real soil property across the landscape. Such a soil dataset provided a quasi-

126 unlimited number of pseudo-measured sites that made probabilistic sampling (and therefore

$127 \mathrm{ME}$ and MSE variance calculations) applicable without any effect on the predictive

128 performances. It also enabled the calculation of any uncertainty indicators from their

empirical distributions obtained by repeating the validation process $\mathrm{n}$ times, which means each time.
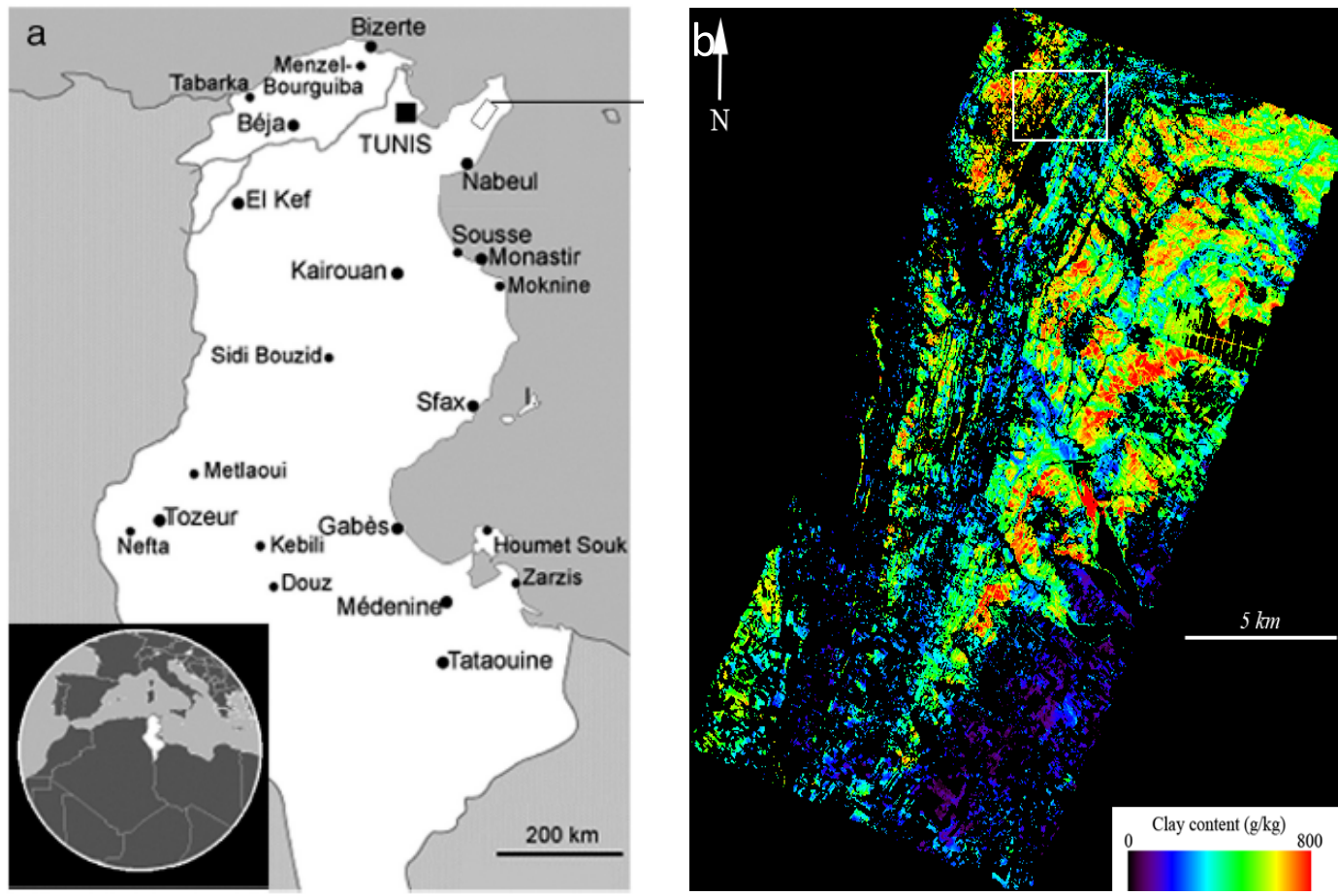

Figure 1: Location of the study area (a) and the spatial pattern of pseudo values of topsoil clay content 134 (b) 


\section{Material and methods}

137

140 The study area is in the Cap Bon region in northern Tunisia $\left(36^{\circ} 24^{\prime} \mathrm{N}\right.$ to $36^{\circ} 53^{\prime} \mathrm{N} ; 10^{\circ} 20^{\prime} \mathrm{E}$ to $14110^{\circ} 58^{\prime} \mathrm{E}$ ), which is $60 \mathrm{~km}$ east of Tunis (Figure 1a). This $300 \mathrm{~km}^{2}$ area includes the Lebna catchment, which is mainly rural $(>90 \%)$. The Lebna catchment is devoted to the cultivation

143 of cereals in addition to legumes, olive trees, vineyards and natural vegetation for animals.

144 The region is characterized by its rolling hills and elevations between 0 and $226 \mathrm{~m}$. The climate

145 varies from humid to semi-arid, with an inter-annual precipitation of $600 \mathrm{~mm}$ and an inter-

146 annual potential evapotranspiration of $1500 \mathrm{~mm}$. The soil pattern of the Lebna catchment is

147 mainly the result of variations in lithology. The variations in the bedrock between Miocene

148 sandstone and Marl cause large variations in the physical and chemical soil properties (Zante

149 et al., 2005). Furthermore, the distance between successive sandstone outcrops decreases 150 significantly as the terrain changes from the ocean to the mountains, which also causes 151 variations in the soil property patterns (Gomez et al., 2012b). The soil materials were 152 redistributed laterally along the slopes during the Holocene, which adds to the complexity of 153 the soil pattern. The main soil types are regosols (IUSS working group WRB, 2006)), which are 154 preferentially associated with sandstone outcrops, and calcic cambisols and vertisols, which preferentially formed on marl outcrops and lowlands. The southeastern region of the study area has a flatter landscape with sandy Pliocene deposits in which calcosols and rendzinas prevail 


\subsection{Data}

2.2.1. Hyperspectral image and derived topsoil clay content predictions

The numerical experiment uses an image of topsoil clay content as input. The data were derived from a Vis-NIR-SWIR hyperspectral image (Gomez et al., 2012b). The approach used to produce the data is summarized below. More details about the pre- and post-processing of the hyperspectral image can be found in Gomez et al (2012b).

On November 2, 2010, AISA-Dual airborne-based hyperspectral data were acquired over the study area with a spatial resolution of $5 \mathrm{~m}$. The area of the image is approximately $12 \mathrm{~km} \times 24$ $\mathrm{km}$. The AISA-Dual spectrometer measured the reflected radiance via 359 non-contiguous bands covering the 400 to $2450 \mathrm{~nm}$ spectral range, with $4.6 \mathrm{~nm}$ bandwidths between 400 and $970 \mathrm{~nm}$ and $6.5 \mathrm{~nm}$ bandwidths between 970 and $2450 \mathrm{~nm}$. The instantaneous field of view (IFOV) was 24 degrees. Topographical corrections were performed using a digital elevation model built from ASTER data and ground control points.

To isolate the bare soil areas, the study masked pixels with normalized difference vegetation index (NDVI) values greater than an expert-calibrated threshold (0.20). Water and Urban areas were also removed. Finally, the bare soil represented $46.3 \%$ of our study area and potentially $5,889,847$ measured AISA-Dual $5 \mathrm{~m} \times 5$ m pixels.

A Partial Least Square Regression (PLSR) technique (Tenenhaus, 1998) was then applied to estimate the topsoil clay contents from the 280 reflectance bands provided by the AISA-DUAL airborne sensor at each location. The PLSR was calibrated from 129 couples of Vis-NIR-SWIR reflectance spectra acquired by the AISA-DUAL sensor on bare soil surfaces associated with the topsoil clay content measured on a laboratory soil sample collected from the same bare 
soil surfaces. Before the PLSR model was built, the reflectance was converted into "absorbance" (log [1/reflectance]). In addition, a Savitzky-Golay filter with second-order polynomial smoothing and window widths of $30 \mathrm{~nm}$ (Savitzky and Golay, 1964) and a mean 186 centering and variance scaling was applied to the spectra to reduce noise. The calibrated PLSR 187 model was then validated using a leave-one-out cross-validation that showed successful 188 predictions $\left(R^{2}=0.75\right)$. The PLSR model was then applied to all bare soil pixels to estimate the 189 topsoil clay content, thus providing the final predicted topsoil clay properties map (Figure 1b), 190 which is denoted "pseudo values of Clay content". These treatments were implemented in R 191 (Version 1.17) using the signal and pls packages (Mevik and Wehrens, 2007).

A 30-m ASTER digital elevation model (DEM) with specific ortho-rectification and mosaicking was produced for this area. The classical geomorphometric indicators found in the DSM 197 literature were calculated. These include Elevation, Slope, Aspect, plan Curvature, Profile Curvature and Multi-Resolution Valley Bottom Flatness (MRVBF). Sine and cosine 199 transformations were applied to the 'aspect' to obtain four indices with a continuous gradient: 'northness', 'easterness','north-westerness' and 'north-easterness'. Finally, the $X$ and $Y$ coordinates (the n of "scorpan" in McBratney et al., 2003) were also used as soil covariates.

2.3. Checking the plausibility of the predicted soil patterns

205 We conducted a prior check to confirm the reliability of using the pseudo values of the clay content determined above as a realistic example of a soil property pattern. It was particularly 
important to check the absence of any distortion of the spatial pattern due to the spectral

208

209

210

211

212

213

215

216

217

measurements by remote sensing. Three experimental variograms showing the spatial structure of clay variations were calculated and then fitted with an exponential model using the weighted least square method (Cressie, 1993) using three different data sets: i) the 129 sites with laboratory measurements of the clay content, ii) the same sites with pseudo values of the clay content and iii) 100,000 randomly selected sites with pseudo values of the clay content. Comparisons of the variograms were performed (see section 3.1.).

\subsection{Sampling technique}

We used probability sampling for selecting the calibration set used to build the DSM model, evaluating the performance of these models and undertaking the numerical experiment. All of the probability sampling techniques followed the same sampling approach (the stratified random sampling technique) using compact geographical strata (Walvoort et al., 2010) recommended by Brus et al. (2011). The main advantage of this technique is that it ensures an even distribution of the samples over the studied area and is simple to apply.

The stratified random sampling approach was applied as follows. The study area was first stratified into 25 geographical strata of equal area using a K-means classification of the $\mathrm{X}$ and $\mathrm{Y}$ coordinates of each locations. We then randomly selected pixels of the grid within each stratum with a fixed number of locations in accordance with the total of samples required.

\subsection{Uncertainty indicators}


We considered four uncertainty indicators among the possible ones that could have been

231 examined. The first two were the mean error (ME) and the mean squared error (MSE) that 232 were selected because these are the classical indicators whose variance can be calculated 233 from analytical expressions (Brus et al, 2011). The last two were the mean square error skill 234 score (SS mse) (Wilks, 2011 p 359, cited by NussBaum et al, 2017)) and the Prediction Interval 235 Coverage probability (PICP) (Shrestha and Solomatine 2006). SS $_{\text {MSE }}$ is similar to the $\mathrm{R}^{2}$ reported 236 in some studies (Vaysse and Lagacherie, 2015, Viscarra-Rossel, 2015) as the percentage of 237 variance explained by the model:

$$
S S_{m s e}=1-\frac{\sum_{i=1}^{i=n}\left(z_{i}-z_{i}^{*}\right)^{2}}{\sum_{i=1}^{i=n}\left(z_{i}-\widehat{\bar{z}}\right)^{2}}
$$

239 Where $z_{i}$ and $z_{i}^{*}$ are the respective observed and predicted values of property $z$ at location I, 240 and $\widehat{\overline{z_{l}}}$ is the mean value of $z$.

241 The PICP expresses the probability that all observed values fit within the $90 \%$ prediction limits 242 provided by the DSM model (see section 2.7.1.).

243 It must be noted that the first three uncertainty indicators (ME, MSE and SSMSE) are 244 measurements of the accuracy of predictions, whereas PICP is a measurement of the accuracy 245 of the uncertainty prediction.

246 The calculations of these indicators should take into account the fact that a stratified random 247 sampling technique is applied for selecting the independent sites. Following Brus et al (2011), 248 these calculations are as follows.

249 The estimations of the ME, MSE, SS MSE and PICP correspond to a global mean that was 250 estimated by design-based inference, particularly by the usual estimator for stratified random 251 sampling.

$$
\hat{\bar{y}}=\sum_{h=1}^{H} w_{h} \hat{\bar{y}}_{h}
$$




$$
\hat{\bar{y}}_{h}=\frac{1}{n_{h}} \sum_{i=1}^{n_{h}} y_{h i}
$$

254 Where $H$ is total number of strata $(H=25), w_{h}$ is the weight of stratum $h$ quantified by the

255 relative area, $\hat{\bar{y}}_{h}$ is the estimated mean of the stratum $h, n_{h}$ is the number of sampling points 256 in stratum $h$, and $y_{h i}$ is the measurement of the indicator at location $i$ in stratum $h$. For the $257 \mathrm{ME}, y_{h i}$ can be replaced by the difference between the actual value $z_{h i}$ and the 258 prediction $z^{*}{ }_{h i}$. The MSE can be estimated by replacing $y_{h i}$ with the squared difference 259 between the actual value $z_{h i}$ and the prediction $z^{*}{ }_{h i}$. The SS MSE and the PICP can be estimated 260 by replacing $y_{h i}$ by the SS MSE and the PICP of the stratum $h$.

261

262

2.6. Analytical calculations of standard errors of ME and MSE

263 If probabilistic sampling is applied, it is possible to calculate the standard errors of the ME and 264 MSE. In the case of a stratified random sampling, the equations are (from De Gruijter, 2006)

$$
\widehat{S E}(\bar{y})=\sqrt{\sum_{h=1}^{H} a_{h}^{2} \widehat{V}\left(\hat{\bar{y}}_{h}\right)}
$$

Where $\widehat{V}\left(\hat{\bar{y}}_{h}\right)$ is the sampling variance of the stratum mean $\hat{\bar{y}}_{h}$, which is estimated by :

$$
\begin{aligned}
& \hat{V}\left(\hat{\bar{y}}_{h}\right)=\frac{s_{h}^{2}}{n_{h}} \\
& s_{h}^{2}=\frac{1}{n_{h}-1} \sum_{i=1}^{n_{h}}\left(y_{h i}-\hat{\bar{y}}_{h}\right)^{2}(6)
\end{aligned}
$$

\subsection{DSM modelling}

274 Since the validation process tested in this paper is model free, any model used in DSM could have been selected as an example of a DSM model. However, only two criteria were 
considered: i) the model had to provide local uncertainty predictions for being able to test

277 PICP, and ii) the model should be run without manual intervention and repeated a great 278 number of times in the numerical experiment. We combine these two criteria results in selecting the Quantile Regression Forest as the example DSM model.

This section describes Random Forests and Quantile Random Forests. More details on these two machine learning algorithms are given in the seminal papers by Breiman et al (2001) and by Meinshausen (2006), respectively.

Let $Y$ be a real-valued response variable and $X$ be a covariate or predictor variable that is likely high-dimensional. A standard goal of statistical analysis is to infer the relationship between $Y$ and $X$. Random Forests grow a large $(>500)$ ensemble of trees using $n$ independent observations $\left(Y_{i}, X_{i}\right), i=1, \ldots, n$. Each tree grows via a recursive partitioning of the source set using one predictor variable $X$. At each step, the source set is split into two subsets following a test on the value of $X$. When $Y$ is a quantitative variable, the selected test is the one that minimizes the within subset variance of $Y$ (Breiman et al., 1984). The recursive partitioning is 293 limited by a stopping rule, and the subsets are produced by the last split being the leaves of the tree. The ensemble of trees is produced by using a random sample of the training data and a random subset of the predictor variables for each tree. $n$ : 
302 where $w_{\theta i}(x, \theta)$ is the weight vector given by a positive constant that is one if the observation $303 \quad Y_{i}$ is part of the same leaf and is 0 otherwise.

304 By using Random Forests, the prediction is the average prediction of $k$ single trees that were constructed as described above.

$$
\begin{aligned}
\widehat{Y_{T}}(x) & =\sum_{i=1}^{n} w_{T i}(x) Y_{i} \\
\text { With } \quad w_{T i}(x) & =k^{-1} \sum_{t=1}^{k} w_{\theta i}(x, \theta)
\end{aligned}
$$

One could assume that the weighted observations deliver a good approximation not only of the conditional mean but also of the full conditional distribution. This assumption is at the heart of the Quantile Regression Forest algorithm, which estimates the conditional distribution function of $Y$ given $x$ via

From this conditional distribution, it is possible to derive both the predicted value (the mean) and the bound of the $90 \%$ prediction interval that predicts the associated uncertainty (the 0.05 and 0.95 quantiles).

$$
\hat{F}(y \mid x)=\sum_{i=1}^{n} w_{i}(x) 1_{\left\{Y_{i} \leq y\right\}}
$$


323 values of clay content. The locations were selected according to the stratified sampling

324 techniques described above. The increasing number of sites was selected to obtain contrasted

325 predictive performances (see section 3.2.)

326 After removing the calibration sites, we selected a master evaluation set of 100,000

327 independent sites by applying the stratified random sampling technique using the compact

328 geographical strata described in section 2.5. The reference values of the three uncertainty

329 indicators of interest were computed from this master evaluation set. This set was then

330 removed from the set of possible sites to ensure the independence of the further numerical

331 experiment.

332

333 2.8. Empirical simulation

334

335 The empirical simulation aims to evaluate the amount of variation in the four uncertainty

336 indicators (ME, MSE, SS MSE and PICP) when different evaluation sets are selected. This

337 variation can then be used as an estimate of the uncertainty caused by relying on the choice

338 of a single specific evaluation set, as is always the case in reality.

339 The empirical simulation proceeds as follows:

340 1. Sample a set of $n$ evaluation sites using a stratified random sampling technique using

$341 \quad$ compact geographical strata,

342 2. Calculate the uncertainty indicators over the $n$ sites,

$343 \quad 3 . \quad$ Repeat steps 1 and 2500 times, and

344 4. Compute the distributions and their summary statistics from the 500 values of the uncertainty indicators. 
346 The tested numbers of sites ranged between $n=50$ and $n=500$ with an increment of 25 . This

347 represents the densities of the observations ranging between $1 / 2.67 \mathrm{~km}^{2}$ and $1 / 0.27 \mathrm{~km}^{2}$.

349 2.9. Software

350

351 The software for Random Forests and Quantile Random Forest are made available in R ( $R$

352 Development Core Team, 2008) with the packages RandomForest (Liaw and Wiener, 2002)

353 and quantregForest (Meinshausen and Schiesser, 2015), respectively. Stratified sampling

354 using compact geographical strata is implemented in the R package "spcosa" (Walvoort et al., 355 2010). Variogram studies (section 3) were performed with the gstat package (Pebesma, 2004). 356

$357 \quad 3 . \quad$ Results

358

359 3.1. Check of the predicted soil patterns

360

361 To check the plausibility of using the pseudo values of clay content derived from hyperspectral data, we compared the experimental variograms and fitted model variograms obtained using real clay content measurements at measured sites, the pseudo values of the clay content at 364 the same locations, and a set of 10,000 sites with the pseudo values of the clay content.

Figure 2 shows the experimental and the fitted variograms of the topsoil clay content calculated from different inputs. The parameters of the variogram estimated from the pseudo values of clay content (figure $2 \mathrm{~b}$ ) were similar to those estimated with the real clay content measurements at the same locations (figure 2a). Indeed, the shapes, ranges and sills were 
close to one another. The only noticeable difference was a smaller nugget value exhibited by

370 the variogram of the pseudo values of the clay contents.

371 The experimental variogram obtained from 100,000 sites was much less noisier than but very

372 similar to the previous one.

374 Figure 2: Variograms of clay content obtained from a) 129 sites with clay content laboratory 375 measurements, b) the same 129 sites with pseudo values of clay content c) 100,000 sites with pseudo 376 values of clay content.

\section{Uncertainty indicators}

\begin{tabular}{l|c|ccc}
\hline Number of calibration sites & ME (g/kg) & MSE $\left(\mathrm{g}^{2} / \mathrm{kg}^{2}\right)$ & SS $_{\text {MSE }}$ & PICP (\%) \\
\hline 200 & $-11.3(0.5)$ & $19555(89)$ & 0.29 & 88.5 \\
\hline 2,000 & $-7.0(0.4)$ & $13550(69)$ & 0.51 & 90.3 \\
\hline 50,000 & & & & 0.78 \\
\hline
\end{tabular}



independent sites

Table 1 shows the values of the uncertainty indicators calculated from the master set of 387 independent sites (100,000 sites) for the DSM models obtained by calibrating the quantile regression Forests with three sizes of calibration sets. As expected, the overall accuracy of the measured predictions (as measured by $\mathrm{SS}_{\mathrm{MSE}}$ ) increased significantly as the number of calibration sites increased, while the bias measured by the ME and mean squared error (MSE)

391 decreased. The PICP values were found to be close to the expected value of 90 for the two 392 models with the greatest numbers of calibration sites. Meanwhile, the model built from 200 393 calibration sites exhibited a PICP below 90, which revealed a slight underestimation of the uncertainty. Finally, the results obtained by the three models well covered the large range of performances of DSM models that can be encountered in the literature. It is also worth noting that the standard errors of the ME and MSE that were calculated from the variances given by equations 8 and 9 are very small, which means that the performances of the three models are 398 significantly different from each other.

399 In the following, the QRFs calibrated from 200, 2,000 and 50,000 sites are denoted, 400 respectively, as "low-quality QRF", "medium-quality QRF” and "high-quality QRF". 

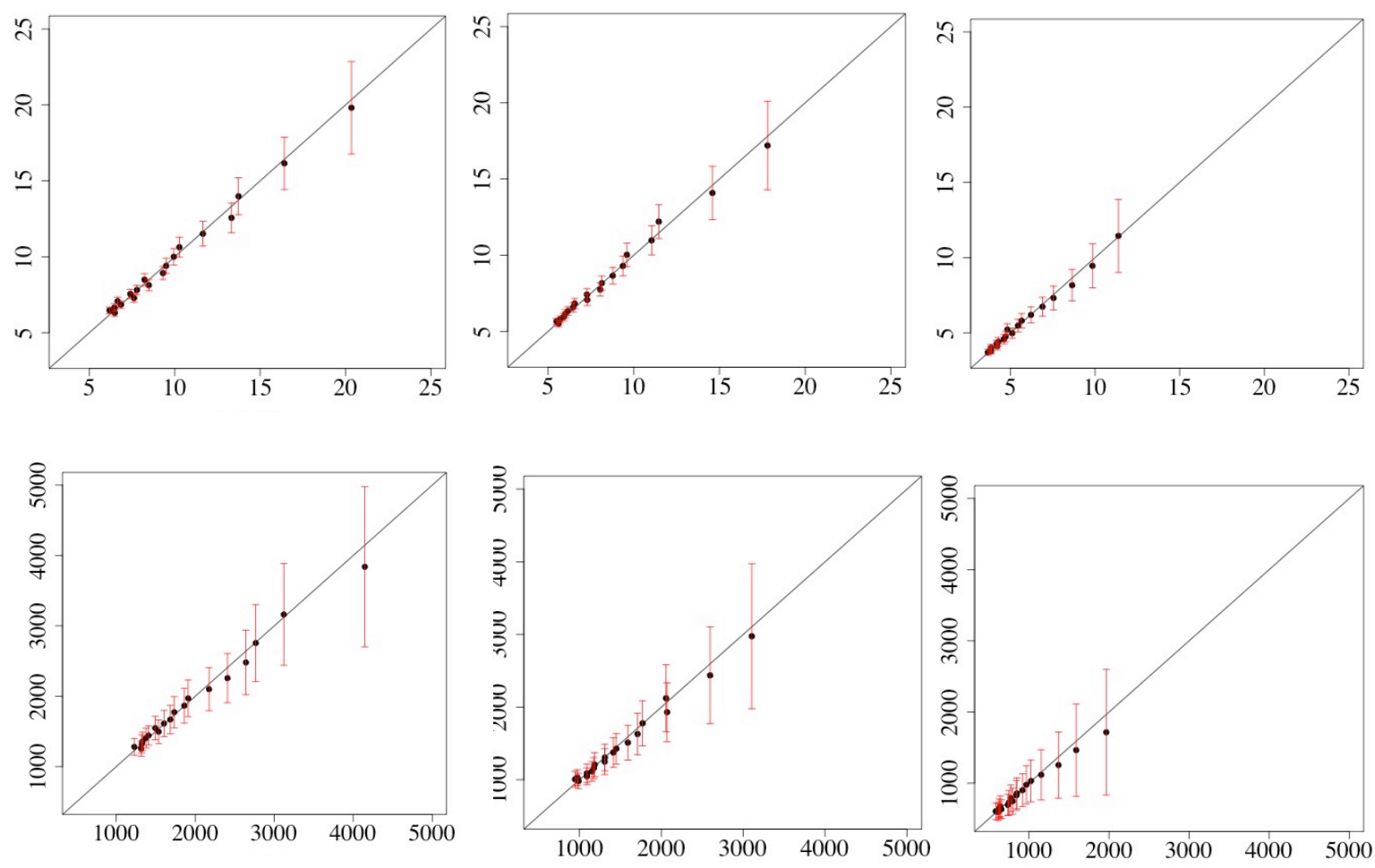

401

402

403

404

405

406

407

408

409

410

411

412

413 The dots of figure 3 representing the mean values over the 500 iterations of the calculated

414 standard errors of the ME and MSE were found to be very close to the 1:1 line. This denoted

good agreement between the analytical calculations of the ME and MSE and the simulation

416

Figure 3: Comparisons between the estimations of standard errors of ME and MSE derived from the numerical experiment ( $\mathrm{X}$-axis) and their analytical calculations ( $\mathrm{Y}$-axis). ME (first row), MSE (second row), low quality QRF (first column), medium quality QRF (second column) and high-quality QRF (third column). Dots : mean values of the calculated standard errors on ME and MSE over the 500 iterations, bars: twice the standard deviations of the calculated standard errors on ME and MSE over the 500 iterations)

\subsection{Comparisons of estimated standard errors of ME and MSE}

For each DSM model, the standard deviations of the ME and MSE observed over the 500

iterations for the different sizes of evaluation sets were compared with the standard errors of the ME and MSE calculated following equations 4, 5 and 6 (figure 3).

outputs, which was expected. Interestingly, the error bars around the dots of figure 3 
417 representing twice the standard deviation over the 500 iterations of the analytical calculations

418 of the standard errors of the ME and MSE were great for the largest standard errors and

419 decreased as the standard errors decreased, regardless of the indicator and the DSM model.

420 This revealed the residual impacts of the evaluation sites' locations that were selected by the

421 spatial sampling, the DSM model and the size of the evaluation set being fixed. 

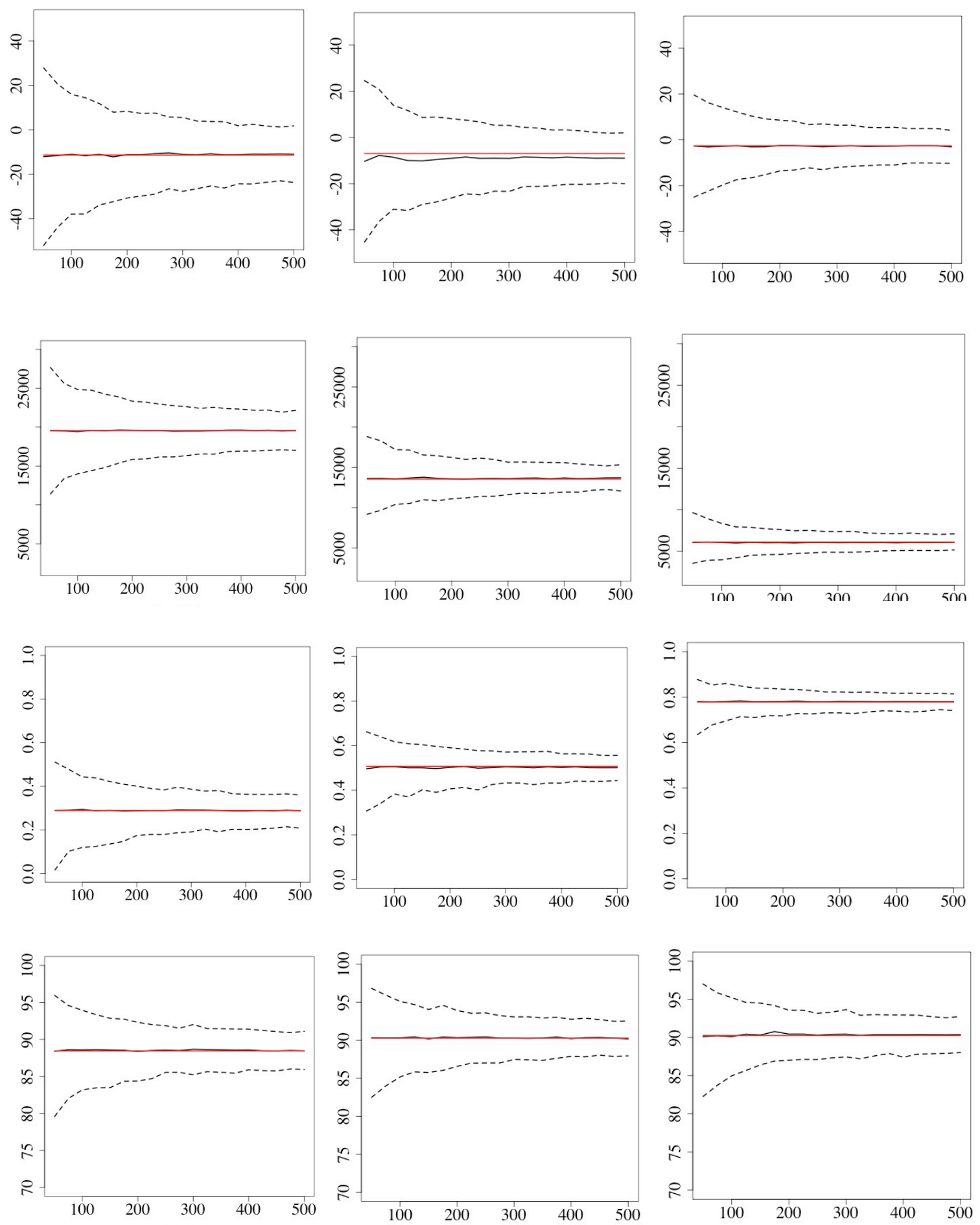

423 Figure 4: Values of uncertainty indicators obtained using validation sets of different sizes (from 50 to

424500 sites): ME (first row), MSE (second row),SS

425 (first column), medium quality QRF (second column) and high-quality QRF (third column). Black line: 

of the uncertainty indicators over 500 trials.. Red line: reference values of the uncertainty indicators

Figure 3 shows the result of the numerical experiment on the three tested QRFs and the four uncertainty indicators. Each result consists of three graphical curves showing the evolution of the median (black line) and the 0.05 and 0.95 quantiles (dotted black lines) of the uncertainty indicator values, with the size of evaluation sets expressed by its number of sites (in abscissa).

434 A red line shows the reference value of the indicator, as calculated from the master evaluation 435 set.

All the results exhibited similar patterns. The mean values of the uncertainty indicators were

437 all close to the reference values. The differences between the quantiles (or the confidence 438 interval widths) were generally large, which reveals imprecise estimations of the uncertainty 439 indicators.

440 An increase in precision was observed as the sizes of the evaluation sets increased. For small 441 sets (fewer than 100 sites), the confidence interval widths were so great that the estimations 442 of the uncertainty indicators were weakly informative. For the largest sets (500 sites), the 443 confidence interval widths were much smaller but still conveyed a non-negligible imprecision. 444 For example, the confidence interval widths for the ME, SS $_{\text {MSE }}$ and PICP were, respectively, 17 $445 \mathrm{~g} / \mathrm{kg}, 0.11$ and $5 \%$ for the medium precision QRF.

446 Some differences in the results across uncertainty indicators and models had to be noted. The 447 high precision QRF exhibited less difference between quantiles than did the two other QRFs 448 for the ME, MSE and SS MSE. A "better" model would also be a model whose performance can 
be more easily assessed. However, this was not the case for PICP, which exhibited differences in quantile values as large as those observed for the two other models.

451

452

453

\section{Discussion}

454

455

\subsection{Suitability of hyperspectral data for testing DSM models}

456

The comparisons of the variograms showed that the pseudo values of the clay content obtained from hyperspectral data represented the spatial structure of a soil property well, apart from a smoothing of the small range variability revealed by a decrease in the nugget value. It must be noted that similar sill and range closeness and nugget decreases were observed in a previous study (Gomez et al, 2012a). This smoothing is attributable to several

462 factors that may perturb the spectral signature of the topsoil clay content (Lagacherie et al., 2008): atmospheric conditions, changes in support (a square block of $5 \mathrm{~m}$ side) compared to

464 soil sampling, or variations in the stoniness, vegetation and rugosity of the soil surface. This may be also the result of using the partial least square regression that as a linear model, smooths the variations of the predicted variable. This slight underestimation of the variability

467 of clay content could lead to slightly underestimated uncertainty indicators. However, this 468 artefact cannot compromise the results obtained from the variations across the sampling of 469 these indicators.

470 The spatial pattern of the pseudo values of clay content (as obtained from hyperspectral data)

471 can be considered a good approximation of a real pattern of soil properties while providing a

472 quasi-unlimited set of possible sites with soil property measurements. In this paper, we exploit 
473 these advantages for experimentally assessing the quality of estimations of the usual

474 uncertainty indicators of DSM models, which, to the best of our knowledge, has not been done

475 before. Furthermore, such data make it possible to accurately validate and compare DSM

476 models thanks to the large size of the validation sets, from which the uncertainty indicators

477 can be computed.

478 Although it cannot be envisaged that spatial sets of pseudo values of soil properties derived

479 from airborne hyperspectral imagery could be collected for each DSM application, several 480 study areas with such soil datasets across the world can be used to enlarge the range of tested 481 soil properties and pedological contexts (Schwangart and Jarmer, 2011; Stevens et al., 2010; 482 Ben Dor et al., 2002, Gomez et al., 2012a, Vaudour et al, 2016). Furthermore, other study 483 areas could be added to this initial set with the aim of building national, regional or global 484 benchmarks for DSM models, which exist in other disciplines (Rosensweig et al., 2013, Luo et 485 al., 2012).

Our results (figure 4) revealed that the uncertainty indicators can vary across evaluations sets, 490 from different sample counts, and between evaluation sets of the same number of samples. 491 This highlights that the uncertainty indicators calculated from statistical validations are 492 themselves (depending on the evaluation setup) prone to non-negligible uncertainty.

493 It therefore can be claimed from these results that too low of a number of evaluation sites 494 cannot accurately estimate the performance of DSM models since the values of the 495 uncertainty indicators may vary a lot with the locations of the evaluation sites. Even with large numbers of evaluation sites and an unbiased probability sampling, there can still be an 
imprecision that prevents the models from being ranked in terms of the calculated uncertainty

498

499

500

501

502

503

504

505

506

507

508

509

510

511

512

513

514

515

516

517 indicators if the differences in their performances are too small. For example, two models with differences in SS MSE below 0.05 may have confidence intervals that overlap each other by more than half their width. However, it must be noted that the ME, MSE and $\mathrm{SS}_{\mathrm{MSE}^{2}}$ calculated for a $^{2}$ "high quality" model could be less uncertain. The decrease in the uncertainty of predictions also correspond with a decrease in the spatial variability of the errors, which in turn may correspond with a decrease of the sensitivity of these uncertainty indicators to the evaluation dataset. The question then is how does one know for sure that a model is of high quality; because of the variability of the indicators for low and average quality models, some models might mistakenly be considered high quality models. This seems particularly true when considering indicators such as PICP and MSE. This emphasizes the usefulness of estimating models using a set of complementary indicators.

\subsection{Benefit of the numerical experiment}

It must be noted that the impact of the sample size on the uncertainty of some uncertainty indicators (ME and MSE) is already well established in analytical expressions (equations 4, 5 and 6) that have been applied in a few DSM studies (e.g. Kempen et al, 2011). As expected, our numerical experiment well reproduced the results obtained from the analytical expressions both for estimating the uncertainty of the ME and MSE (figure 3) and for reproducing the effect of the sample sizes (Figure 4). Beyond reproducing these results, the large number of evaluation procedures performed in a same study $(27,000)$ provided a comprehensive understanding of the respective impacts of the DSM model quality, the number of evaluation sites and the location of these sites on the values of uncertainty

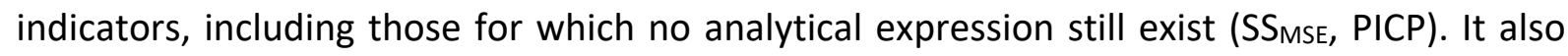


521 revealed that the analytical calculations of the ME and MSE uncertainties were themselves

522 prone to uncertainty that could be important for the evaluation sets having the largest

523 variances (Figure 3), which corresponded to those with the smallest sized evaluation sets

524 (Figure 4).

525 In the future, such a numerical experiment can also be used for obtaining references about 526 the loss of precision in evaluating the uncertainty of DSM models when it is not possible to 527 perform a probabilistic sampling and thus to calculate ME and MSE by the analytical 528 expressions. This occurs in most of the current DSM applications that use legacy soil data

529 whose locations have been selected by a soil surveyor following a non-probabilistic process 530 ("free survey").

531

532 4.4. Improving the evaluation process

533

534 Our results clearly showed that the uncertainty of Digital Soil Mapping products cannot be 535 estimated with a great precision. This must be better taken into account in the practices of

536 soil mapping evaluations. A first recommendation is to systematically assess the standard 537 error of the uncertainty indicators using the available analytical formulations when possible 538 or by bootstrapping the validation set. Furthermore, better attention should be paid to the 539 sampling techniques used to select the evaluation sites. Indeed, stratified random sampling 540 using compact geographical strata ensures an even distribution of sites across space but does 541 not avoid the error on the uncertainty indicators. Reducing this error by using more 542 sophisticated sampling techniques is a priority. In this perspective, our case study provides a

543 quasi-infinite number of validation sets that exhibited differences with the master validation 544 set regarding the values of the uncertainty indicators. Analysing the variability of these 
545 differences would permit the sampling criteria to be found that would ensure more accurate

546 estimations. The new sampling techniques could also be extended to the calibration datasets

547 of DSM models that provide a priori estimations of their errors or that are used for evaluations

548 with cross-validation techniques (Brus et al., 2011). In addition, using uncertainty indicators

549 that are less sensitive to outliers (Nusbaum et al., 2014) would be a complementary way to

550 reduce the uncertainty of the uncertainty revealed by this paper. Finally, although a

551 quantitative assessment of uncertainty represents a great progress over the current

552 evaluation practices of traditional soil surveys, it should be completed by an expert-based

553 assessment that could check the plausibility of the predicted spatial patterns with regard to 554 the available pedological knowledge.

555

556

557

\section{Conclusion}

558

559

Different evaluation sets obtained by probabilistic sampling were tested for their ability to assess the prediction uncertainty of DSM models using (as a case study) a spatial pattern of pseudo-values of topsoil clay content obtained from airborne hyperspectral imagery. The main lessons are summarized as follows:

- The spatial patterns of pseudo-values of some soil properties that could be available in some study areas across the world constitutes a relevant network for experimental assessments of the uncertainty of validation results. This is because i) it allows the DSM model to be evaluated by using many sites that could not be envisaged if only real soil data were used, and ii) it allows different numbers and locations of possible evaluation sets to be tested. Thus, it may provide an useful complement to the analytical 

which these analytical expressions are not valid.

571

572

573

574

575

576

577

578

579

580

581

582

583

584

585

586

587

588

589

- Any evaluation from independent sets conveys a non-negligible error on the uncertainty indicators that is greater when the number of sites is low. Such evaluations should therefore be interpreted with care and the uncertainty on validation results must be systematically estimated.

- The sampling techniques used for the calibration and evaluation datasets should be improved to reduce this error.

\section{Acknowledgments}

This research was conducted within the "Centre d'Expertise Scientifique Cartographie Numérique des sols" granted by the CNES-TOSCA program. We thank David Rossiter and an anonymous referee for their useful comments for improving the paper.

\section{References}

Arrouays, D., McBratney, A.B., Minasny, B., Hempel, J.W., Heuvelink, G.B.M., MacMillan, R.A., Hartemink, A.E., Lagacherie, P., McKenzie, N.J., 2014. The GlobalSoilMap project specifications, in: Arrouays, D., McKenzie, N.J., Hempel, J.W., Richer-de-Forges, A.C., McBratney, A.B. (Eds.), GlobalSoilMap: Basis of the global Spatial soil information system. CRC press \& Taylor \& francis group, Boca Raton, USA, pp 1-12 
590 Ben-Dor E., Patkin K., Banin A., Karnieli A., 2002. Mapping of several soil properties using DAIS5917915 hyperspectral scanner data - a case study over clayey soils in Israel. International 592 Journal of Remote Sensing, 23, p. 1043-1062

593 Breiman, L., Friedman, J., Stone, C.J., Olshen, R.A., 1984. Classification and regression trees. $594 \quad$ CRC press.

595 Breiman, L., 2001. Random Forests. Machine Learning 45, 5-32.

596 Brus, D.J., Kempen, B., Heuvelink, G.B.M., 2011. Sampling for validation of digital soil maps. $597 \quad$ Eur. J. Soil Sci. 62, 394-407.

598 Cho, E, Cho, M.J., Eltinge, J., 2004. The variance of sample variance from a finite population. 599 Proceedings of Joint American Statistical Association and International Statistical Institute 600 Conference Toronto Canada

601

Cochrane, W.G., Snedecor, G.W., 1989. Statistical methods. Eigth edition. Iowa State 602 University Press, Ames (lowa).

603

Cressie, N.J.A., 1993. Statistics for spatial data. Revised edition. Wiley interscience publication. 604 New York.

605

606

607

608

609

610

611

Gomez, C., Coulouma, G., Lagacherie, P., 2012a. Regional predictions of eight common soil properties and their spatial structures from hyperspectral Vis-NIR data, Geoderma, 189$190,176-185$

Gomez, C., Lagacherie P., Bacha, S. 2012b. Using an VNIR/SWIR hyperspectral image to map topsoil properties over bare soil surfaces in the Cap Bon region (Tunisia). In "Digital Soil Assessments and Beyond" Minasny B., Malone B.P., McBratney A.B. (Ed.).Springer, 387392. 
612

626

Gomez, C., Oltra Carrio, R., Lagacherie, P., Bacha, S., and Briottet, X. 2015. Sensitivity of soil property prediction obtained from Hyperspectral Vis-NIR imagery to atmospheric effects and degradation in image spatial resolutions. Remote Sensing of Environment 164, 1-15.

Goovaerts, P., 2001. Geostatistical modeling of uncertainty in soil science. Geoderma 103, 326.

Heuvelink, G.B.M., 2014. Uncertainty quantification of GlobalSoilMap products. in: Arrouays, D., McKenzie, N.J., Hempel, J.W., Richer-de-Forges, A.C., McBratney, A.B. (Eds.), GlobalSoilMap: Basis of the global Spatial soil information system. CRC press \& Taylor \& Francis group, Boca Raton, USA, pp 327-332.

ISSS, ISRIC, FAO, 1998. World Reference Base for Soil Resources. 84 World Soil Resources report. FAO Rome Italy.

IUSS (International Union of Soil Scientists) Working Group WRB , 2006. World Reference Base for Soil Resources 2006, World Soil Resources Report No. 103. Food and Agriculture Organization of the United Nations, Rome, Italy.

Kempen, B., Brus, D.J., Stoorvogel, J.J., 2011. Three-dimensional mapping of soil organic matter content using soil type-specific depth functions. Geoderma 162, 107-123.

Lagacherie, P., Baret, F., Feret, J.-B., Madeira Netto, J., Robbez-Masson, J.M., 2008. Estimation of soil clay and calcium carbonate using laboratory, field and airborne hyperspectral measurements. Remote Sensing of Environment 112, 825-835. DOI 10.1016/j.rse.2007.06.014 
633 Lagacherie, P., Gomez, C., in press. Vis-NIR-SWIR Remote Sensing Products as New Soil Data 634 for Digital Soil Mapping. in pedometrics A.B. McBratney et al. (eds.), Chapter 13. Progress in Soil Science, Springer. DOI 10.1007/978-3-319-63439-5_13

Liaw, A., Wiener, M., 2002. Classification and Regression by randomForest. R News 2(3), 18-22.

Luo, Y.Q., Randerson, J.T., Abramowitz, G., Bacour, C., Blyth, E., Carvalhais, N., Ciais, P., Dalmonech, D., Fisher, J.B., Fisher, R., Friedlingstein, P., Hibbard, K., Hoffman, F., Huntzinger, D., Jones, C.D., Koven, C., Lawrence, D., Li, D.J., Mahecha, M., Niu, S.L., Norby, R., Piao, S.L., Qi, X., Peylin, P., Prentice, I.C., Riley, W., Reichstein, M., Schwalm, C., Wang, Y.P., Xia, J.Y., Zaehle, S., Zhou, X.H., 2012. A framework for benchmarking land models. Biogeosciences 9, 3857-3874.

644

McBratney, A.B., Mendonca Santos, M.L., Minasny, B., 2003. On digital soil mapping. 645 Geoderma 117, 3-52.

646

Meinshausen, N., 2006. Quantile Regression Forests. J. of Machine Learning Res. 7, 983-999.

Meinshausen, N., Schiesser, L., 2015. quantregForest: Quantile Regression Forests. R package.

648 https://cran.r-project.org.

649

Mevik, B.-H., Wehrens, R. (2007); The pls Package: Principal Component and Partial Least 650 Squares Regression in R; Journal of Statistical Software 18(2), 1-24 1210. 
654 Nussbaum, M., Spiess, K., Baltensweiler, A., Grob, U., Keller, A., Greiner, L., Schaepman,

655

656

657

658

659

660

661

662

663

664

665

666

667

668

669

670

671 Stevens, A., Udelhoeven, T., Denis, A., Tychon, B., Lioy, R., Hoffman, L., Van Wesemael, B.,

672

673

674

M.E., Papritz, A., 2017. Evaluation of digital soil mapping approaches with large sets of environmental covariates. SOIL Discuss. 1-32.

Pebesma, E.J., 2004. Multivariable geostatistics in S: the gstat package. Computers \& Geosciences, 30: 683-691.

R Development Core Team (2008). R: A language and environment for statistical computing. R Foundation for Statistical Computing, Vienna, Austria.

Rosenzweig, C., Jones, J.W., Hatfield, J.L., Ruane, A.C., Boote, K.J., Thorburn, P., Antle, J.M., Nelson, G.C., Porter, C., Janssen, S., Asseng, S., Basso, B., Ewert, F., Wallach, D., Baigorria, G., Winter, J.M., 2013. The Agricultural Model Intercomparison and Improvement Project (AgMIP): Protocols and pilot studies. Agric. For. Meteorol. 170, 166-182.

Savitzky, A., \& Golay, M.J.E. (1964). Smoothing and differentiation of data by simplified least squares procedures. Analytical Chemistry, 36(8), 1627-1639

Schwanghart, W., Jarmer, T., 2011. Linking spatial patterns of soil organic carbon to topography - A case study from south-eastern Spain. Geomorphology 126, 252-263.

Shrestha, D.L., Solomatine, D.P., 2006. Machine learning approaches for estimation of prediction interval for the model output. Neural Networks 19 (2), 225-235. 2010. Measuring soil organic carbon in croplands at regional scale using airborne imaging spectroscopy. Geoderma 158, 32-45.

Tenenhaus, M. (1998). La régression PLS. Editions Technip, Paris. 254 pp 
675 Vaudour, E., Gilliot, J.M., Bel, L., Lefevre, J., Chehdi, K., 2016. Regional prediction of soil organic 676 carbon content over temperate croplands using visible near-infrared airborne 677 hyperspectral imagery and synchronous field spectra. Int. J. Appl. Earth Observ. Geoinf., 678 49, 24-38. DOI: 10.1016/j.jag.2016.01.005

679 Walvoort, D.J.J., Brus, D.J., de Gruijter, J.J., 2010. An R package for spatial coverage sampling 680 and random sampling from compact geographical strata by k-means. Computers and $681 \quad$ Geosciences, 36, 1261-1267.

682 Zante, P., Collinet, J., Pepin, Y., 2005. Caractéristiques pédologiques et hydrométéorologiques 683 du bassin versant de Kamech, Cap Bon, Tunisie. UMR LISAH IRD Tunis, DG ACTA Direction 684 des Sols Tunis, INRGREF Tunis. (21 p. +6 annexes).

685

686 